11

\title{
Моделирование нуклеации в бинарных сплавах на основе метода функционала плотности свободной энергии
}

\author{
(C) П.Е. ЛЬвов ${ }^{1,2}$, В.В. Светухин ${ }^{3}$, С.В. Булярский ${ }^{2}$ \\ ${ }^{1}$ Ульяновский государственный университет, \\ Ульяновск, Россия \\ ${ }^{2}$ Институт нанотехнологий микроэлектроники РАН, \\ Москва, Россия \\ ${ }^{3}$ НПК „Технологический центр“, \\ Москва, Зеленоград, Россия \\ E-mail: LvovPE@sv.uven.ru
}

Поступила в Редакцию 16 июля 2019 г.

В окончательной редакции 16 июля 2019 г.

Принята к публикации 25 июля 2019 г.

\begin{abstract}
Разработан подход для расчета скорости зарождения выделений вторых фаз на основе метода функционала плотности свободной энергии. Скорость зарождения, а также характеристики критического зародыша рассчитываются непосредственно из результатов моделирования эволюции концентрационного поля,полученного с помощью решения детерминированного или стохастического уравнения Кана-Хилларда.
\end{abstract}

Ключевые слова: скорость зарождения; метод функционала плотности свободной энергии; уравнение КанаХилларда; бинарные сплавы.

DOI: $10.21883 /$ FTT.2019.12.48597.05ks

\section{1. Введение}

Проблема образования зародышей новой фазы является одной из базовых проблем теории фазовых переходов первого рода, протекающих по механизму зарожденияроста. Данный механизм реализуется в области стабильных и метастабильных состояний и требует преодоления барьера зародышеобразования, связанного с формированием устойчивых зародышей $[1,2]$.

Основной характеристикой формирования зародышей является скорость зародышеобразования или скорость нуклеации $I_{C}^{*}$, которая определяется как количество устойчивых зародышей, сформированных в единицу времени в единице объема [2]:

$$
I_{C}^{*}=\frac{d X_{C}^{*}}{d t}
$$

где $X_{C}^{*}$ - количество зародышей в единице объема, размер которых превышает критическое значение $R>R^{*}$. Здесь и далее под размером будем понимать эффективный радиус зародыша, который связан с количеством находящихся в нем атомов $N_{C}$ в виде $R=\left(3 N_{C} v_{0} /(4 \pi)\right)^{1 / 3}$, $v_{0}$ - объем, приходящийся на один атом.

Также на практике может быть использована „измеряемая“ скорость нуклеации, которая равна производной от общего числа наблюдаемых зародышей $X_{C}[2]$ : $I_{C}=\frac{d X_{C}}{d t}$. Использование данной характеристики может быть обусловлено рядом факторов [2]. Во-первых, зародыши с размером больше или меньше критического неотличимы друг от друга. Во-вторых, чувствительность используемых приборов может не позволить зафиксировать критические зародыши ввиду малости их размера.
Расчет скорости зарождения обычно проводится на основе известных подходов теории нуклеации $[1,2]$. Одной из наиболее распространенных является запись нестационарной скорости зародышеобразования в виде [1]:

$$
I_{C}^{*}=I_{0}^{*} \exp \left(-\frac{\Delta G^{*}}{k_{\mathrm{B}} T}\right) \exp \left(-\frac{t_{i}}{t}\right),
$$

где $\Delta G^{*}$ - работа образования зародыша критического размера, которая обычно считается постоянной величиной, $t_{i}-$ инкубационное время, $t-$ время, $T-$ температура, $k_{\mathrm{B}}$ - постоянная Больцмана. Расчетные соотношения для предэкспоненциального множителя $I_{0}^{*}$ и инкубационного времени $t_{i}$, а также их зависимости от концентрации мономеров и температуры могут существенно отличаться в зависимости от используемых моделей [1].

В классической теории нуклеации работа образования критического зародыша со сферической геометрией определяется на основе капиллярного приближения и может быть вычислена с помощью соотношения [1,2]: $\Delta G^{*}=\frac{4 \pi}{3} R^{* 2} \sigma$, где $\sigma-$ поверхностное натяжение на границе между зародышем и матрицей.

В случае формирования наноразмерных выделений вторых фаз ширина переходного слоя между ядром зародыша и матрицей оказывается сравнимой с размером самого зародыша, а состав может существенно отличаться от соответствующих макроскопических фаз. В этом случае разделение объемной и поверхностной части энергии зародыша оказывается невозможным. Задача о расчете работы формирования таких зародышей может быть решена на основе метода функционала плотности 
свободной энергии [1-3]. Рассматривая зародыш с помощью функционала Гинзбурга-Ландау, можно получить соотношение для расчета энергии сферического зародыша критического размера, помещенного в однородную безграничную матрицу $[1,3]$ :

$$
\Delta G^{*}=4 \pi n_{0} \int_{0}^{\infty}\left(g(c)-\frac{c-c_{M}}{2} \frac{\partial g}{\partial c}\right) r^{2} d r,
$$

где $g(c)$ - свободная энергии смешения в расчете на одну частицу, $c_{M}-$ средний состав сплава, $r-$ радиусвектор, $n_{0}$ - количество атомов в единице объема.

В действительности проблема определения энергии образования зародышей может оказаться более сложной задачей, поскольку они формируются в матрице, которая является сильно неоднородной ввиду наличия тепловых флуктуаций. На ранней стадии фазового перехода формирующиеся зародыши, как устойчивые, так и неустойчивые, могут взаимодействовать между собой, что также влияет на процесс преодоления барьера зародышеобразования. Кроме того, в процессе фазового перехода происходит изменение степени пересыщения вещества в матрицы, что приводит к изменению условий зародышеобразования.

В связи с этим возникает задача о расчете работы и скорости зародышеобразования непосредственно из результатов моделирования фазового перехода на основе детерминированного или стохастического уравнения Кана-Хилларда, которые позволяют естественным образом учитывать перечисленные выше особенности.

\section{2. Моделирование зародышеобразования с помощью детерминированного уравнения Кана-Хилларда}

Уравнение Кана-Хилларда, полученное в работе [4]:

$$
\frac{\partial c}{\partial t}=M \nabla^{2}\left(\frac{\partial g}{\partial c}-\kappa \nabla^{2} c\right)
$$

широко известно своим применением к фазовым переходам, протекающим по механизму спинодального распада. Здесь $M-$ подвижность $(M=$ const $), g \equiv g(c)-$ свободная энергия смешения в расчете на один атом, для которого примем приближение регулярных растворов $[1,3], \kappa-$ коэффициент градиентной энергии $(\kappa=$ const). Данное уравнение может быть использовано также для описания фазовых переходов в области стабильных и метастабильных состояний, протекающих по механизму нуклеации $[1,2]$ вблизи границы устойчивости $[5,6]$.

Поскольку в области стабильных и метастабильных состояний для образования вторых фаз требуется преодоление барьера зародышеобразования, то для применения детерминированного уравнения КанаХилларда (4) в данной области существенное значение имеет формулировка начального условия. Данное начальное условие может быть сформулировано на основе теории равновесных флуктуаций [7] с использованием длинноволнового приближения [5].

Граничные условия считались периодическими. Решение уравнения Кана-Хилларда осуществлялось с помощью полуявного спектрального метода [8]. Идентификация зародышей выполнялась на основе метода ближайшего соседа [5].

На рис. 1 приведен пример решения уравнения Кана-Хилларда (4) для трехмерного фрагмента бинарного сплава с размером $256 a \times 256 a \times 256 a$. Для данной системы очевидно наличие стадий: зарождения (рис. $1, a, b)$, роста (рис. $1, b, c)$ и коалесценции (рис. $1, c, d)$. На основе анализа эволюции концентрационного поля рассчитана измеряемая скорость зарождения $I_{C}=d X_{C} / d t$, которая отражена на рис. 2. Очевидно, что данная скорость зарождения характеризуется инкубационным временем. Затем происходит достаточно быстрый рост концентрации обнаруженных зародышей $X_{C}$, и скорость зарождения достигает своего максимального значения. Далее скорость зарождения начинает снижаться и достигает нуля. Это обусловлено истощением матрицы по атомам растворенного компонента, что приводит к росту барьера нуклеации и невозможности формирования новых зародышей. На рисунках время $\tau$ и температура $T^{*}$ приводятся в безразмерных единицах: $\tau=t \cdot 2 M k_{\mathrm{B}} T_{C} / a^{2}$ и $T^{*}=T / T_{C}$, где $a-$ период решетки, $T_{C}=\Omega /\left(2 k_{\mathrm{B}}\right)$ - критическая температура регулярного раствора, связанная с параметром взаимодействия $\Omega$.

Одним из основных факторов протекания фазового перехода является наличие пересыщения, поэтому важно установить связь между скоростью зарождения $I_{C}$ и степенью пересыщения. Как следует из рис. 3, данная зависимость может быть удовлетворительно аппроксимирована степенной или линейной зависимостью. На рисунке скорость зарождения приводится в указанных безразмерных единицах.

\section{3. Расчет скорости зародышеобразования с помощью уравнения Кана-Хилларда-Кука}

Наиболее полное рассмотрение процесса образования зародышей по механизму нуклеации может быть выполнено на основе стохастического уравнения КанаХилларда (уравнение Кана-Хилларда-Кука) [9], которое дает возможность непосредственного учета тепловых флуктуаций

$$
\frac{\partial c}{\partial t}=M \nabla^{2}\left(\frac{\partial g}{\partial c}-\kappa \nabla^{2} c\right)+\sqrt{\varepsilon} \xi
$$

где $\varepsilon-$ постоянный безразмерный коэффициент порядка единицы, $\xi$ - случайная сила, описываемая с помощью флуктуационно-диссипационной теоремы [9]. 

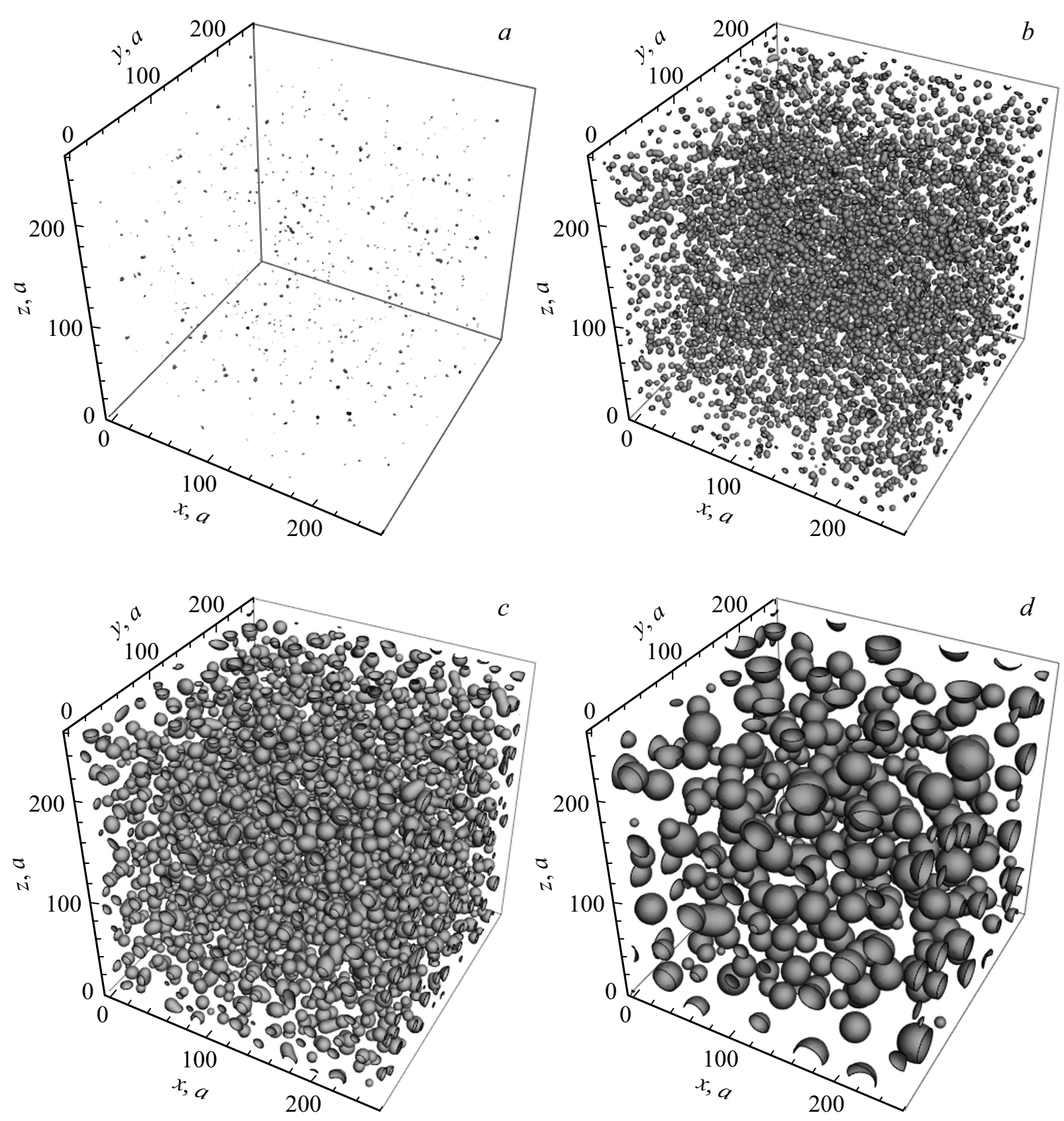

Рис. 1. Различные стадии фазового перехода в бинарном сплаве с постоянной подвижностью и составом $c_{M}=16$ at\% при температуре $T^{*}=0.65: a, b-$ зарождение; $b, c$ - рост; $c, d-$ коалесценция. Представленные распределения фаз соответствуют различным моментам времени $\tau: 0.1,20,370$ и 5120. Границы раздела фаз показаны поверхностями постоянной концентрации $(c=50$ at $\%)$.

Применение уравнения (5) для описания процесса нуклеации необходимо выполнять также в длинноволновом приближении, которое обусловлено атомной природой вещества [9], а также используемым формализмом концентрационного поля, для расчета которого в каждой точке системы требуется анализировать от нескольких десятков до нескольких сотен узлов решетки [5]. Данная особенность учитывается путем введения параметра обрезания $k_{\max }$, для которого компоненты Фурье-образа концентрационного поля $\hat{c}_{\mathbf{k}} \equiv 0$ при $|\mathbf{k}|>k_{\max }[6,10]$.
Значение $k_{\max }$ может быть также связано с корреляционной длиной [9].

В случае сильных флуктуаций возможно формирование существенно-неравновесных функций распределения по размерам, что приводит к искажению кинетики среднего размера и концентрации [6]. В отдельных случаях сильные флуктуации могут также приводить к сдвигу критической температуры [7] и, как следствие, отсутствию фазового перехода [6] (см. также [11]). 


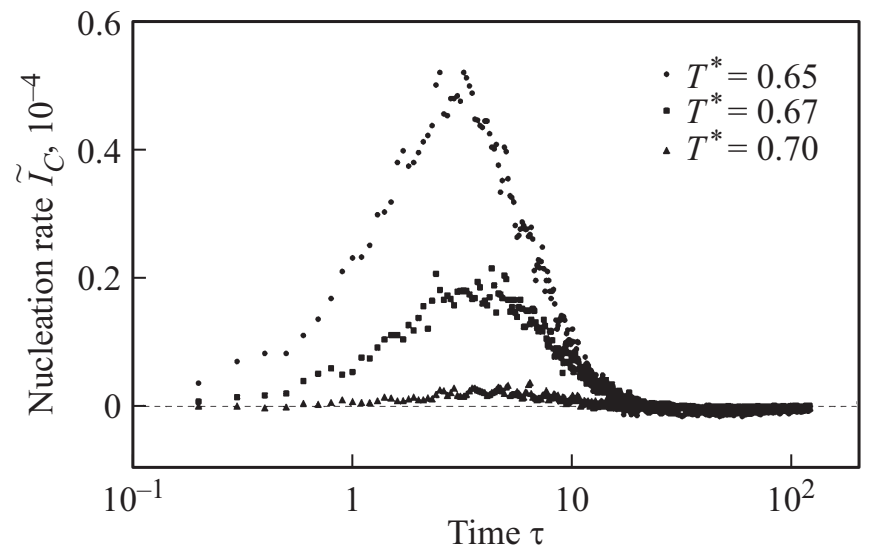

Рис. 2. Зависимость измеряемой скорости зарождения $\tilde{I}_{C}=I_{C} v_{0} a^{2} /\left(2 k_{\mathrm{B}} T M\right)$ от времени $\tau$ в сплаве с составом $c_{M}=16$ at\% для различных температур $T^{*}: 0.65,0.67$ и 0.7 .

Для расчета скорости нуклеации необходимо определить параметры критического зародыша, что может быть сделано с использованием следующего алгоритма. Вопервых, должна быть рассчитана эволюция концентрационного поля на ранней стадии распада с помощью уравнения (5) [6,10]. Затем необходимо вычислить работу формирования в расчете на одну частицу для всех наблюдаемых зародышей по формуле $[1,3,10]$ :

$$
\begin{aligned}
\bar{W}_{i} & =\frac{n_{0}}{N_{i}} \int_{V_{i}}\left(g(c)+\kappa(\nabla c)^{2}\right) d V \\
& \approx \frac{n_{0}}{N_{i}} \int_{V_{i}}\left(g(c)-\frac{c-c_{M}}{2} \frac{\partial g}{\partial c}\right) d V
\end{aligned}
$$

в различные моменты времени. Здесь $V_{i}-$ объем, занимаемый зародышем с номером $i, N_{i}=n_{0} V_{i}-$ количество атомов в зародыше. При этом $V_{i}$ необходимо выбирать таким образом, чтобы он полностью охватывал переходный слой между матрицей и ядром зародыша. Далее строится зависимость $\bar{W}_{i}$ от числа атомов $N_{i}$ и среднего состава зародыша $\bar{c}_{i}$. Максимальное значение работы формирования $\bar{W}^{*}=\max _{i} \bar{W}_{i}-$ соответствует искомым значениям параметров критического зародыша: работе зародышеобразования $\Delta G^{*}=N^{*} \bar{W}^{*}$, размеру зародыша $R^{*}$ и его среднему составу $\bar{c}^{*}$.

Пример использования данного подхода к определению параметров критического зародыша приведен на рис. 4 для $\varepsilon=0.25, k_{\max }=1.2 a^{-1}$ и размера области моделирования $256 a \times 256 a \times 512 a$. Как следует из рисунка, рассматриваемые зависимости работы формирования зародыша от размера и среднего состава имеют выраженные максимумы. Соответствующие значения параметров критического зародыша, определенные по рис. 4 составляют: $\bar{W}^{*} \sim 0.029 k_{\mathrm{B}} T_{C}, R^{*} \sim 3.2 a$, $\bar{c} \sim 45$ at\%. Рассчитанное значение работы зародышеобразования согласуется с расчетами, полученными с помощью решения стационарного уравнения КанаХилларда [3]: $\bar{W}^{*} \sim 0.031 k_{\mathrm{B}} T_{C}[10]$.

Таким образом, определяя параметры критического зародыша можно установить скорость нуклеации в виде (1), где в качестве $X_{C}^{*}$ следует принять концентрацию зародышей, имеющих размер и состав больше полученных критических значений: $R>R_{C}^{*}, \bar{c}>\bar{c}^{*}$.

По аналогии с рис. 3 аппроксимируем скорость нуклеации с помощью линейной зависимости от степе-
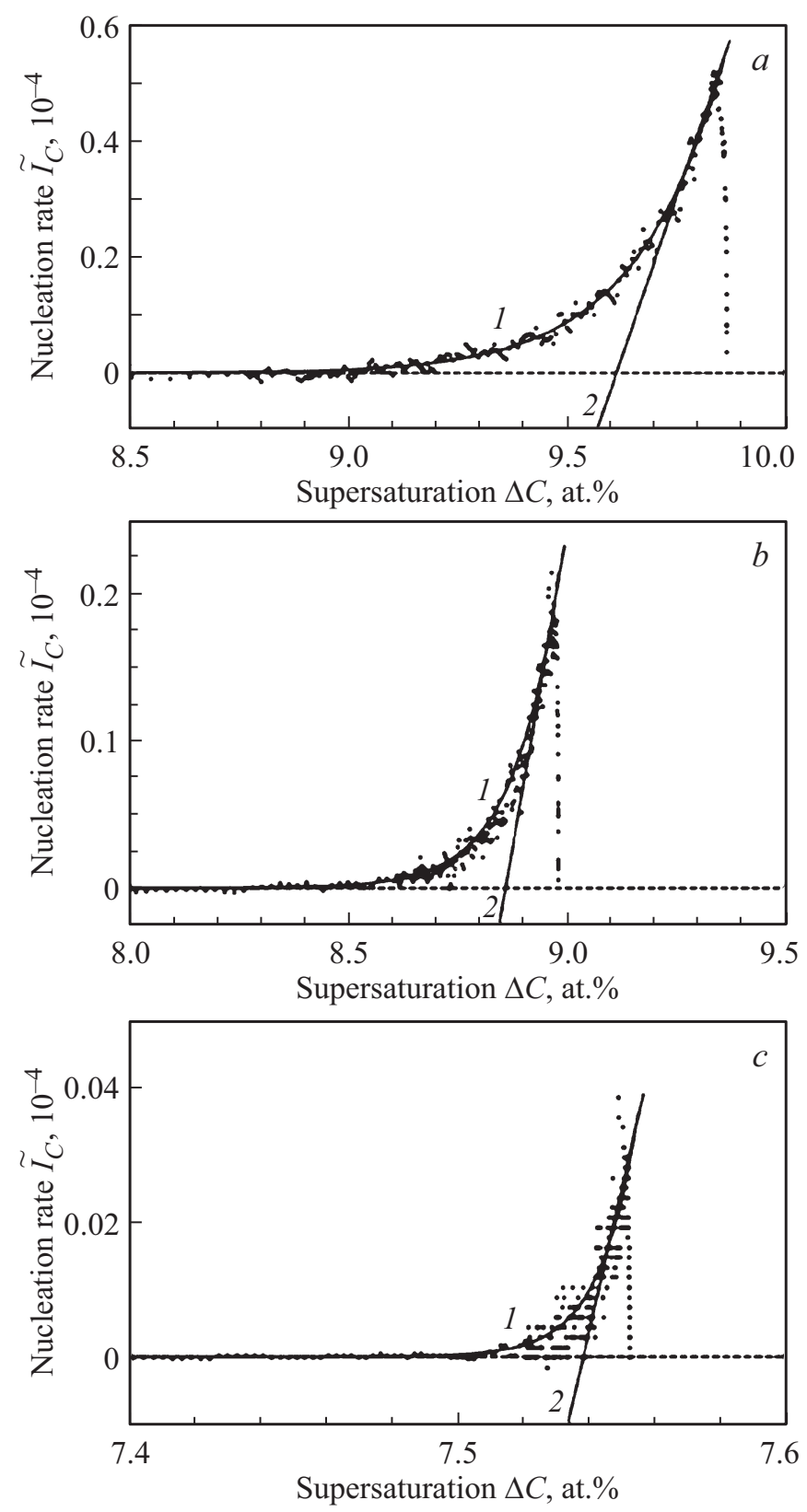

Рис. 3. Зависимость измеряемой скорости зарождения $\tilde{I}_{C}=I_{C} v_{0} a^{2} /\left(2 k_{B} T M\right)$ от степени пересыщения $\Delta c$ в сплаве с составом $c_{M}=16$ at\% для различных температур $T^{*}: a-$ $T^{*}=0.65, b-T^{*}=0.67, c-T^{*}=0.7$. Точки - результат решения уравнения (4). Сплошные линии: 1 - степенная аппроксимация $\left(a-\tilde{I}_{C} \approx \Delta c^{49.06}, b-\tilde{I}_{C} \approx \Delta c^{86.38}, c-\right.$ $\left.\tilde{I}_{C} \approx \Delta c^{656.80}\right), 2$ - линейная аппроксимация. 
ни пересыщения (см. также [5]). Учитывая наличие инкубационного времени в виде (2), можно получить удобную формулу для приближенного расчета скорости нестационарной нуклеации

$$
I_{C}^{*}=I_{0}^{*}\left(C_{M}-C_{I}^{*}\right) \exp \left(-\frac{t_{i}}{t}\right),
$$

где $C_{M}-$ среднее значение состава матрицы, зависящее от времени $t, C_{I}^{*}-$ равновесный состав матрицы над поверхностью критического зародыша. Определение постоянных параметров $t_{i}$ и $I_{0}^{*}$ может быть выполнено путем сравнения скорости формирования зародышей, полученной с помощью (7), и результатов решения уравнения (5). Пример определения параметров скорости зарождения приведен на рис. $5, a, b$ (см. также [10]). Параметры скорости зародышеобразования в (7) составили: $\tilde{I}_{0}^{*}=0.5 \cdot 10^{-4}, \tau_{i}=164.9$ для $c_{M}=12$ at $\% ; \tilde{I}_{0}^{*}=8.5 \cdot 10^{-4}, \tau_{i}=134.2$ для $c_{M}=13 \mathrm{at} \%$; $\tilde{I}_{0}^{*}=28 \cdot 10^{-4}, \tau_{i}=116.9$ для $c_{M}=14 \mathrm{at} \%$. Как и ожидалось, увеличение концентрации растворенного компонента при неизменной температуре приводит к росту предэкспоненциального множителя $\tilde{I}_{0}^{*}$ и уменьшению инкубационного времени $\tau_{i}$.
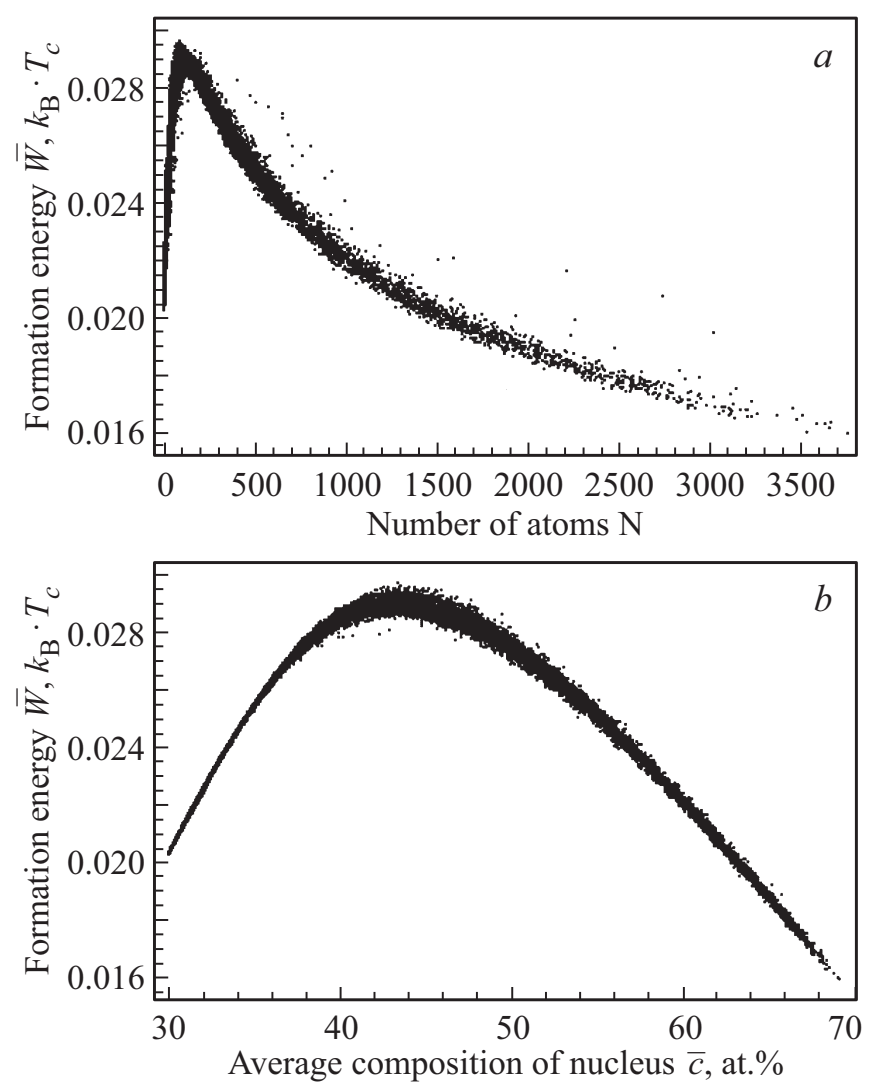

Рис. 4. К определению характеристик критического зародыша с помощью уравнения Кана-Хилларда-Кука в сплаве $c_{M}=12$ at $\%$ при $T^{*}=0.6896, \varepsilon=0.25, k_{\max }=1.2 a^{-1}$. Показана зависимость работы формирования зародыша $\bar{W}$ от количества атомов в зародыше $(a)$, а также от состава зародыша $(b)$.
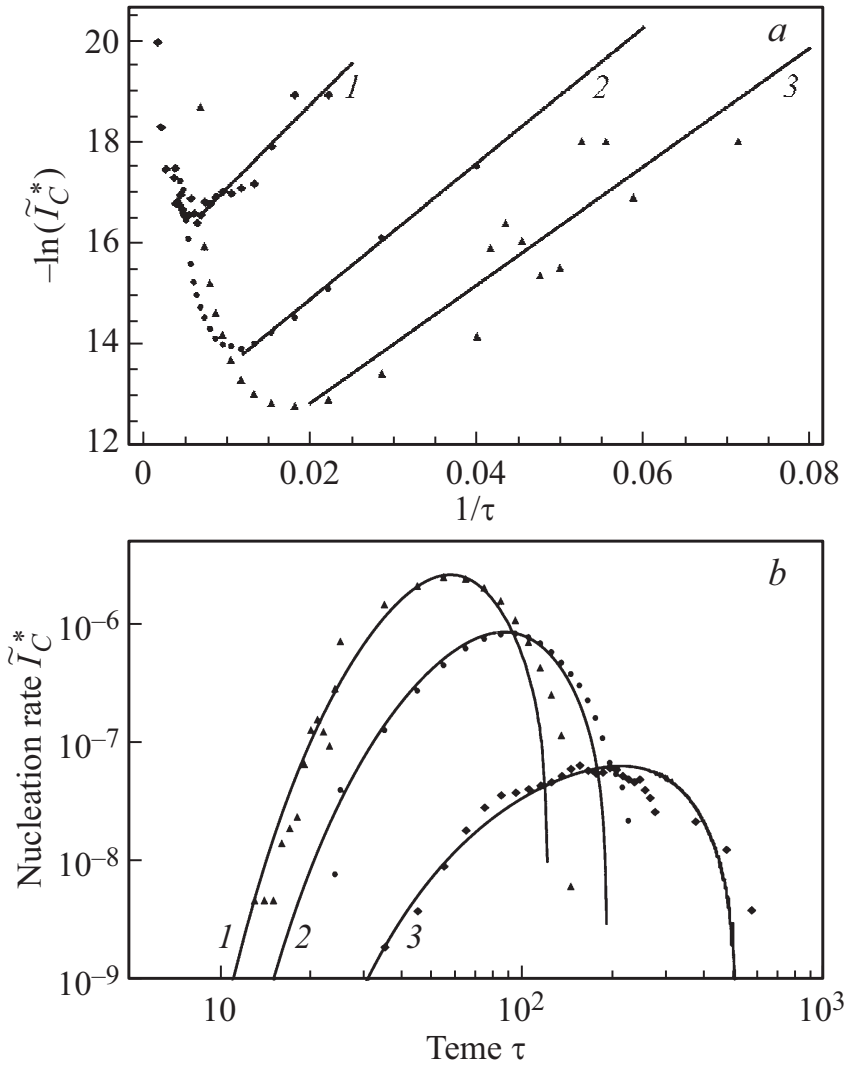

Рис. 5. Зависимость скорости нуклеации в безразмерных единицах $\tilde{I}_{C}^{*}=I_{C}^{*} v_{0} a^{2} /\left(2 k_{B} T M\right)$ от времени $\tau$ при температуре $T^{*}=0.6896$ для сплавов с составами: $1-c_{M}=12$ at\%, $2-$ $c_{M}=13 \mathrm{at} \%, 3-c_{M}=14 \mathrm{at} \%$. $a-$ определение параметров аппроксимации $\tau_{i}$ и $\tilde{I}_{0}^{*} . b-$ результаты расчета зависимости скорости нуклеации $\tilde{I}_{C}^{*}$ от времени [10].

Разработанный подход по определению скорости зарождения и полученная аппроксимация (7) может быть использована для построения системы кинетических уравнений, описывающих фазовый переход как блуждание закритических зародышей в пространстве размеров $[10,12]$. При этом кинетические коэффициенты определяются с помощью детерминированного или стохастического уравнения Кана-Хилларда вместо уравнения нормальной диффузии, используемого в классической теории нуклеации.

\section{4. Выводы}

В работе предложен подход к анализу ранней стадии фазового перехода первого рода, который позволяет рассчитывать характеристики критического зародыша (размер, состав, работу формирования) и скорость зарождения непосредственно из решения детерминированного или стохастического уравнения Кана-Хилларда. Расчет данных величин может быть выполнен при известных параметрах взаимодействия между компонентами систе- 
мы, использование каких-либо дополнительных приближений не требуется.

\section{Финансирование работы}

Работа выполнена при финансовой поддержке Министерства науки и высшего образования (проект № 00042019-0001), Российского фонда фундаментальных исследований и Правительства Ульяновской области (проект № 18-42-732002).

\section{Конфликт интересов}

Авторы заявляют об отсутствии конфликта интересов.

\section{Список литературы}

[1] H. Aaronson, M. Enomoto, J. Lee. Mechanisms of Diffusional Phase Transformations in Metals and Alloys. CRC Press, Taylor \& Francis Group, Boca Raton. (2010). 667 p.

[2] D. Kashchiev. Nucleation. Basic Theory with Applications. Butterworth-Heinemann: Eastbourne. (2000). 551 p.

[3] J.W. Cahn, J.E. Hilliard. J. Chem. Phys. 31, 688 (1959).

[4] J. Cahn. Acta Met. 9, 795 (1961).

[5] P.E. L'vov, V.V. Svetukhin. Modelling Sim. Mater. Sci. Eng. 25, 075006 (2017).

[6] P.E. L'vov, V.V. Svetukhin. Modelling Sim. Mater. Sci. Eng. 26, 045001 (2018).

[7] Л.Д. Ландау, Е.М. Лифшиц. Статистическая физика. Ч. 1. Наука. М. (1976). 586 c.

[8] L.-Q. Chen, J. Shen. Comput. Phys. Commun. 108, 147 (1998).

[9] P.C. Hohenberg, B.I. Halperin. Rev. Mod. Phys. 49, 435 (1977).

[10] P.E. L'vov, V.V. Svetukhin. Modelling Sim. Mater. Sci. Eng. 27, 025002 (2019).

[11] J. Fan, M. Sammalkorpi, M. Haataja. Phys. Rev. E 81, 011908 (2010).

[12] П.Е. Львов, В.В. Светухин. ФТТ 57, 1192 (2015).

Редактор Ю.Э. Китаев 